\title{
Triangular numbers whose sum of divisors is also triangular
}

by

Douglas E. IAnnucci (St. Thomas, VI) and Florian LuCa (Morelia)

1. Introduction. For every positive integer $n$ we put $\phi(n)$ and $\sigma(n)$ for the Euler function of $n$ and the sum of divisors function of $n$, respectively. We also put $\phi(0)=\sigma(0)=1$. Let $f(X), g(X) \in \mathbb{Z}[X]$ be polynomials of degrees $>1$ which are integer valued and with positive leading coefficients. In this paper, we study the equations $\phi(f(n))=g(m)$ and $\sigma(f(n))=g(m)$ in positive integers $m$ and $n$. For a positive real number $x$, we put

$$
\begin{aligned}
\mathcal{F}_{f, g}(x) & :=\{n \leq x: \phi(|f(n)|)=|g(m)| \text { for some integer } m\}, \\
\mathcal{S}_{f, g}(x) & :=\{n \leq x: \sigma(|f(n)|)=|g(m)| \text { for some integer } m\} .
\end{aligned}
$$

Our main result consists in an upper bound for the counting functions of the set $\# \mathcal{F}_{f, g}(x)$ and $\# \mathcal{S}_{f, g}(x)$.

THEOREM 1. Assume that $f$ and $g$ are integer-valued, have positive leading coefficients and are of degrees $\operatorname{deg} g \geq \operatorname{deg} f>1$. Assume further that $f$ factors completely over $\mathbb{Q}$ and has only simple roots. Then there exists a positive constant $c:=c(f, g)$ such that

$$
\max \left\{\# \mathcal{F}_{f, g}(x), \# \mathcal{S}_{f, g}(x)\right\} \leq c \frac{x}{(\ln x)^{1 / 10}}
$$

for all $x>e$. Here, $\ln x$ denotes the natural logarithm of $x$.

Note that we have excluded the case when $\operatorname{deg} f=1$. We will comment on this in the final section of the paper.

The conditions that $f(X)$ factors over $\mathbb{Q}$ and has only simple roots are perhaps not needed if one only wants that both $\# \mathcal{F}_{f, g}(x)$ and $\# \mathcal{S}_{f, g}(x)$ are $o(x)$ as $x$ tends to infinity. We have not been able to prove such a statement. Further, the exponent $1 / 10$ on the right hand side of (1) is perhaps not optimal. However, note that if both $p$ and $p+2$ are prime numbers, then $\sigma(p(p+2))=(p+1)(p+3)$. Thus, such a twin prime $p$ leads to

2000 Mathematics Subject Classification: Primary 11N37; Secondary 11A25, 11N36.

Key words and phrases: sum of divisors, Euler function. 
the solution $(n, m)=(p, p+1)$ for the equation $\sigma(f(n))=f(m)$, where $f(n)=n(n+2)$. Since it is believed that the number of twin primes $p \leq x$ is at least $c_{0} x /(\log x)^{2}$, it seems the exponent $1 / 10$ cannot be improved to an exponent larger than 2. Similar considerations apply when $d=\operatorname{deg} f$ is fixed, for which one can use the Prime $k$-tuples Conjecture to construct polynomials $f$ and $g$ of the required degree $d$ for which $\mathcal{F}_{f, g}(x)$ and $\mathcal{S}_{f, g}(x)$ have orders of magnitude at least $x /(\log x)^{d}$. When one also fixes $d_{1}=\operatorname{deg} g>d$, one can use instead Schinzel's Hypothesis $H$ to construct some polynomials $f$ and $g$ of the required degrees for which one can get some large conjectural lower bounds on the cardinalities of the above sets.

To simplify the presentation of our proof, we will illustrate it with details only for the case $f(X)=X(X+1) / 2, g(X)=X(X+1)$ and the set $\mathcal{S}=\mathcal{S}_{f, g}$ and only point out the minor changes one needs to make in order to obtain the full conclusion of Theorem 1. While most "counting" results which hold for the Euler function also hold for the sum of divisors function, it is in general easier to work with the Euler function instead of the sum of divisors function. It is exactly for this reason that we have chosen to treat the "harder" case. Further, this choice of polynomials is not accidental, since every even perfect number is triangular, so it leads naturally to a solution of the equation $\sigma(n(n+1) / 2)=m(m+1)$ with $m=n$ (recall that a number $k$ is perfect if $\sigma(k)=2 k)$.

Throughout this paper, we use the Vinogradov symbols $\gg$ and $\ll$ with their regular meaning. We recall that $U \ll V$ and $V \gg U$ are each equivalent to the assertion that $U=O(V)$.

For a positive real number $x$, we use $\log x$ for the maximum between the natural logarithm of $x$ (denoted $\ln x)$ and 1 , and $\pi(x)$ for the number of primes $p \leq x$. For a positive integer $n$, we write $P(n), p(n), \omega(n), \Omega(n)$ and $\tau(n)$ for the largest prime factor of $n$, the smallest prime factor of $n$, the number of distinct prime divisors of $n$, the number of prime power divisors $(>1)$ of $n$, and the total number of divisors of $n$, respectively. Here, $P(1)=p(1)=\tau(1)=1$ and $\omega(1)=\Omega(1)=0$.

Acknowledgements. We would like to thank an anonymous referee not only for a very careful reading of a first draft of this paper but also for explicit suggestions on how to simplify parts of our original arguments. Research of F. L. was carried out while the author was in residence at the Tata Institute of Fundamental Research in Mumbai, India in November and December of 2005. He thanks the people of that Institute for their hospitality and the Third World Academy of Sciences for support.

2. The proof. We will proceed by eliminating several sets of positive integers $n \leq x$ whose counting function is at least as small as the right hand 
side of estimate (1). Only then do we study the left-over numbers $n \leq x$ in $\mathcal{S}$. We assume that $x$ is a large positive real number.

2.1. Eliminating small integers. Writing

$$
\mathcal{S}_{1}:=\left\{n \leq x /(\log x)^{2}\right\},
$$

we certainly have

$$
\# \mathcal{S}_{1} \leq x /(\log x)^{2} .
$$

From now on, we assume that $n \leq x$ is not in $\mathcal{S}_{1}$.

2.2. Eliminating smooth integers. We put

$$
y:=y(x)=\exp \left(\frac{\log x}{4 \log \log x}\right) .
$$

For a positive integer $n$ we write $P(n)$ for the largest prime factor of $n$ with the convention that $P(1)=1$. It is well-known (see Chapter III of [5]) that if we put

$$
\Psi(x, y):=\#\{n \leq x: P(n) \leq y\}
$$

then

$$
\Psi(x, y) \ll x \exp (-u / 2)
$$

uniformly in $2 \leq y \leq x$, where $u=\log x / \log y$. For us, $u=4 \log \log x$, therefore if we write

$$
\mathcal{S}_{2}:=\{n \leq x: P(n) \leq y \text { or } P(n+1) \leq y\},
$$

then

$$
\# \mathcal{S}_{2} \leq 2 \Psi(x, y)+1 \ll x \exp (-2 \log \log x)=x /(\log x)^{2} .
$$

From now on, we assume that $n \leq x$ is not in $\mathcal{S}_{1} \cup \mathcal{S}_{2}$. In particular, we have $\min \{P(n), P(n+1)\}>y$.

2.3. Eliminating integers with a large powerful divisor. Let $v:=(\log x)^{4}$. Recall that a positive integer $m$ is called powerful if $p^{2} \mid m$ whenever $p$ is a prime factor of $m$. Let

$$
\mathcal{S}_{3}:=\{n \leq x: d \mid n \text { or } d \mid n+1 \text { for some powerful } d>v\} .
$$

For each powerful $d \leq x+1$, there are at most $2(x+1) / d+1 \leq 3(x+1) / d$ positive integers $n \leq x+1$ such that $n \equiv-1,0(\bmod d)$. Thus,

$$
\# \mathcal{S}_{3} \ll \sum_{\substack{d \geq v \\ d \text { powerful }}} \frac{x+1}{d} \ll x \sum_{\substack{d>v \\ d \text { powerful }}} \frac{1}{d} \ll \frac{x}{v^{1 / 2}}=\frac{x}{(\log x)^{2}},
$$

where the last estimate above follows easily by partial summation from the fact that the counting function of the set of powerful numbers up to $t$ is $O\left(t^{1 / 2}\right)$ (see, for example, Theorem 14.4 in [3]). 
From now on, we assume that $n \leq x$ is not in $\mathcal{S}_{1} \cup \mathcal{S}_{2} \cup \mathcal{S}_{3}$. In particular, since $y>v$ for large $x$, we get $P(n) \| n$ and $P(n+1) \| n+1$.

In what follows, we will use Mertens's estimate

$$
\sum_{p \leq t} \frac{1}{p}=\log \log t+A+O\left(\frac{1}{\log t}\right)
$$

which holds for all $t>1$ with some constant $A$, as well as the BrunTitchmarsh estimate

$$
\sum_{\substack{p \leq t \\ p \equiv a(\bmod b)}} \frac{1}{p} \ll \frac{1}{p_{a, b}}+\frac{\log \log t}{\phi(b)},
$$

which holds uniformly for $1 \leq a \leq b \leq t$ and $a$ and $b$ coprime, where $p_{a, b}$ is the first prime congruent to $a$ modulo $b$.

2.4. Eliminating integers whose sum of divisors has too many divisors. Let $K:=\lfloor 10 \log \log x\rfloor$. Recall that for a positive integer $n$ we write $\tau(n)$ for the number of divisors of $n$. Let

$$
\mathcal{B}:=\left\{n \leq x: n \notin \bigcup_{i=1}^{3} \mathcal{S}_{i} \text { and } \tau(\sigma(n))>\exp \left(4 K^{2}\right)\right\} .
$$

To bound $\# \mathcal{B}$, assume first that $n \leq x$ is such that $\omega(n)>K$. Let $\mathcal{B}_{1}$ be the subset of $\mathcal{B}$ consisting of such $n$. Let $m \mid n$ be such that $\omega(m)=K$. Then the number of $n \leq x$ which are multiples of $m$ is $\lfloor x / m\rfloor \leq x / m$. Thus,

$$
\begin{aligned}
\# \mathcal{B}_{1} & \leq \sum_{\substack{m \leq x \\
\omega(m)=K}} \frac{x}{m} \leq x \sum_{\substack{m \leq x \\
\omega(m)=K}} \frac{1}{m} \leq \frac{x}{K !}\left(\sum_{p^{\alpha} \leq x} \frac{1}{p^{\alpha}}\right)^{K} \\
& \leq x\left(\frac{e \log \log x+O(1)}{K}\right)^{K} \ll \frac{x}{(\log x)^{c_{1}}}<\frac{x}{(\log x)^{3}},
\end{aligned}
$$

where $c_{1}:=10 \ln (10 / e)>3$. In the above estimates, we used the multinomial formula, estimate (8), as well as the Stirling formula.

Now let $\mathcal{B}_{2}$ be the set of $n \leq x$ such that there exists a prime $p \mid n$ with $\omega(p+1)>K$. Let $n \in \mathcal{B}_{2}$. Write $n=p m$ for some prime $p \leq x / m$ such that $\Omega(p+1)>K$. Note that $p+1 \leq x / m+1 \leq 2 x / m$. By Exercise 0.5 on page 12 in [2] (which has been worked out explicitly as Lemma 13 in [4]), the number of positive integers $l \leq 2 x / m$ such that $\Omega(l) \geq K$ is

$$
\ll \frac{K}{2^{K}} \frac{x \log \log x}{m} .
$$

In particular, the number of numbers $l \leq x / m$ of the form $p+1$ with $\Omega(l) \geq K$ does not exceed the above bound. Summing up over all $m \leq x$, 
we get

$$
\begin{aligned}
\# \mathcal{B}_{2} & \leq \frac{K x \log \log x}{2^{K}} \sum_{m \leq x} \frac{1}{m} \ll \frac{x \log x(\log \log x)^{2}}{2^{10 \log \log x}} \\
& =\frac{x}{(\log x)^{c_{2}+o(1)}} \ll \frac{x}{(\log x)^{2}},
\end{aligned}
$$

where $c_{2}:=10 \ln 2-1>3$.

Now let $\mathcal{B}_{3}$ be the set of remaining $n \leq x$. Write $n=l m$, where $l$ is powerful and $m$ is squarefree. Then $l$ and $m$ are coprime, so $\sigma(n)=$ $\sigma(l) \sigma(m)$. Thus,

$$
\tau(\sigma(n)) \leq \tau(\sigma(l)) \tau(\sigma(m)) .
$$

Note that $\Omega(l) \leq K$ (because $n$ is not in $\mathcal{B}_{1}$ ), and if $p^{a} \| u$, then $p^{a} \leq v=$ $(\log x)^{4}$, because $n$ is not in $\mathcal{S}_{3}$. Hence,

$$
\sigma(l)=\prod_{p^{a} \| l}\left(p^{a}+p^{a-1}+\cdots+1\right) \leq \prod_{p^{a} \| l}\left(2 p^{a}\right) \leq(2 v)^{K}<\exp \left(K^{2}\right),
$$

and so

$$
\tau(\sigma(l)) \leq \sigma(l) \leq \exp \left(K^{2}\right) .
$$

Furthermore, since $n \notin \mathcal{B}_{1} \cup \mathcal{B}_{2}$, we also have

$$
\tau(\sigma(m))=\tau\left(\prod_{p \mid m}(p+1)\right) \leq 2^{\sum_{p \mid m} \Omega(p+1)} \leq 2^{K^{2}}<\exp \left(K^{2}\right),
$$

and now inequalities (13) and (14) together with (12) show that $\mathcal{B} \subseteq \mathcal{B}_{1} \cup \mathcal{B}_{2}$. Thus, if we put

$$
\mathcal{S}_{4}:=\{n \leq x: n \in \mathcal{B} \cup 2 \mathcal{B} \text { or } n+1 \in \mathcal{B} \cup 2 \mathcal{B}\},
$$

then

$$
\# \mathcal{S}_{4} \leq 4 \# \mathcal{B} \ll \frac{x}{(\log x)^{2}}
$$

From now on, we assume that $n \notin \mathcal{S}_{1} \cup \mathcal{S}_{2} \cup \mathcal{S}_{3} \cup \mathcal{S}_{4}$.

2.5. Eliminating integers whose sum of divisors has too few prime divisors. We put

$$
w:=\exp \left((\log x)^{1 / 4}\right) \quad \text { and } \quad N:=\left\lfloor(24)^{-3}(\log \log x)^{2}\right\rfloor .
$$

For a positive integer $n$ and an interval $\mathcal{I}$ we put

$$
\omega_{\mathcal{I}}(n):=\sum_{\substack{p \in \mathcal{I} \\ p \mid n}} 1
$$

for the number of prime factors $p$ of $n$ which belong to $\mathcal{I}$. 
We now put $\mathcal{I}:=\left[v^{2}, w\right)$ and

$$
\mathcal{C}:=\left\{n \leq x: n \notin \bigcup_{i=1}^{4} \mathcal{S}_{i} \text { and } \omega_{\mathcal{I}}(\sigma(n))<N\right\}
$$

The purpose of this section is to bound $\# \mathcal{C}$. We start by putting

$$
z:=\exp (\sqrt{\log x}) \text { and } R:=\left\lfloor(12)^{-1} \log \log x\right\rfloor,
$$

and letting

$$
\mathcal{C}_{1}:=\left\{n \leq x: n \notin \mathcal{S}_{2} \text { and } \omega_{(z, \infty)}(n)<R\right\} .
$$

Assume that $n \in \mathcal{C}_{1}$. Write $n=P m$, where $P=P(n)>y$ because $n \notin \mathcal{S}_{2}$. Fix $m$. Then the number of possibilities for $P \leq x / m$ is

$$
\pi\left(\frac{x}{m}\right) \leq \frac{x}{m \log (x / m)} \leq \frac{x}{m \log y}=\frac{x \log \log x}{m \log x},
$$

where we have used the fact that $x / m \geq P>y$. Since $n \in \mathcal{C}_{1}$, it follows that we can write $m=m_{1} m_{2}$, where $p\left(m_{1}\right)>z$ and $\omega\left(m_{1}\right)<R$, and $P\left(m_{2}\right) \leq z$. Here $p(m)$ is the smallest prime factor of the positive integer $m$. Summing up estimate (17) over all such choices for $m$, we get

$$
\# \mathcal{C}_{1} \leq \frac{x \log \log x}{\log x}\left(\sum_{\substack{p\left(m_{1}\right)>z \\ \omega\left(m_{1}\right)<R}} \frac{1}{m_{1}}\right)\left(\sum_{P\left(m_{2}\right) \leq z} \frac{1}{m_{2}}\right)
$$

For the first sum in the product (18), we have, again by the multinomial formula, Mertens's estimate (8), and Stirling's formula,

$$
\begin{aligned}
\sum_{\substack{p\left(m_{1}\right)>z \\
\omega\left(m_{1}\right)<R}} \frac{1}{m_{1}} & \leq \sum_{k<R} \frac{1}{k !}\left(\sum_{z<p \leq x} \sum_{\alpha \geq 1} \frac{1}{p^{\alpha}}\right)^{k} \\
& \leq \sum_{k<R} \frac{(\log \log x-\log \log z+O(1))^{k}}{k !} \leq \sum_{k<R}\left(\frac{e \log \log z+c_{3}}{k}\right)^{k},
\end{aligned}
$$

where $c_{3}>0$ is some constant. In the above estimate, we also used the fact that $\log \log x=2 \log \log z$. Since for a fixed positive value of $t$ the function $s \mapsto(e t / s)^{s}$ is increasing for all positive $s<t$, and since $R \leq$ $(12)^{-1} \log \log x<0.5 \log \log x=\log \log z$, it follows easily that in the last sum in (19) the last term dominates, so we get

$$
\sum_{\substack{p\left(m_{1}\right)>z \\ \omega\left(m_{1}\right)<R}} \frac{1}{m_{1}} \ll R\left(\frac{0.5 e \log \log x+c_{2}}{R}\right)^{R} \ll(\log x)^{c_{4}} \log \log x,
$$

where $c_{4}:=(12)^{-1} \log (6 e)<1 / 4$. 
As for the second sum on the right hand side of (18), we have, by Mertens's estimate,

$$
\sum_{\substack{l \leq x \\ P(\bar{l}) \leq z}} \frac{1}{l} \leq \prod_{q \leq z}\left(1-\frac{1}{q}\right)^{-1} \ll \log z=(\log x)^{1 / 2} .
$$

Using estimates (20) and (21) in (18), we get

$$
\# \mathcal{C}_{1} \ll \frac{x(\log \log x)^{2}}{(\log x)^{1 / 2-c_{4}}}<\frac{x}{(\log x)^{1 / 4}}
$$

for large values of $x$ because $c_{4}<1 / 4$.

We now let

$$
f(t):=\exp \left((\log t)^{1 / 4}\right), \quad g(t):=(\log t)^{8}, \quad S(t):=\left\lfloor(24)^{-2} \log \log t\right\rfloor,
$$

put $\mathcal{I}(p):=[g(p), f(p))$ and define the set of primes

$$
\mathcal{P}:=\left\{p: \omega_{\mathcal{I}(p)}(p+1)<S(p)\right\} .
$$

We first obtain a nontrivial upper bound for the counting function of the set $\mathcal{P}(t):=\mathcal{P} \cap[1, t]$. Let

$$
y(t):=\exp \left(\frac{\log t}{4 \log \log t}\right),
$$

and put

$$
\mathcal{P}_{1}(t):=\{p \in \mathcal{P}: p \leq t / \log t\} \cup\{p \in \mathcal{P}: P(p+1) \leq y(t)\} .
$$

The arguments used in Section 2.2 show that

$$
\# \mathcal{P}_{1}(t) \leq \pi(t / \log t)+\Psi(t, y(t)) \ll t /(\log t)^{2} .
$$

From now on, we look at the elements of $\mathcal{P}_{2}(t):=\mathcal{P}(t) \backslash \mathcal{P}_{1}(t)$. Let $p$ be such a prime. Write $p+1=P m$, where $P=P(p+1)>y(t)$. Fix $m$. Then, by Brun's sieve, the number of primes $P \leq(t+1) / m$ such that $P m-1=p$ is also prime is

$$
\ll \frac{t+1}{\phi(m)(\log ((t+1) / m))^{2}} \ll \frac{t}{\phi(m)(\log (y(t)))^{2}} \ll \frac{t(\log \log t)^{3}}{m(\log t)^{2}},
$$

where in the above estimates we have used, aside from the fact that $(t+1) / m \geq P \geq y(t)$, also the minimal order of the Euler function in the interval $[1, t]$, which is $\phi(m) / m \gg \log \log t$. We now write $m=d_{1} d_{2} d_{3}$, where $P\left(d_{1}\right)<g(t), P\left(d_{2}\right)<f\left(t /(\log t)^{2}\right)$ and $p\left(d_{2}\right) \geq g(t)$, and $p\left(d_{3}\right) \geq$ $f\left(t /(\log t)^{2}\right)$. Since $p \in \mathcal{P}_{2}(t)$, we have $p>t /(\log t)^{2}$, therefore $P\left(d_{2}\right)<$ $f\left(t /(\log t)^{2}\right)<f(p)$, while $p\left(d_{2}\right) \geq g(t) \geq g(p)$, so $\omega\left(d_{2}\right)<S(p) \leq S(t)$. Summing up estimates (25) over all the positive integers $m$ of the above 
form, we get

$$
\begin{aligned}
\# \mathcal{P}_{2}(t) \ll & \frac{t(\log \log t)^{3}}{(\log t)^{2}}\left(\sum_{P\left(d_{1}\right) \leq g(t)} \frac{1}{d_{1}}\right) \\
& \times\left(\sum_{\substack{P\left(d_{2}\right) \leq f\left(t /(\log t)^{2}\right) \\
\omega\left(d_{2}\right)<S(t)}} \frac{1}{d}\right)\left(\sum_{\substack{d_{3} \leq t \\
p\left(d_{3}\right) \geq f\left(t /(\log t)^{2}\right)}} \frac{1}{l}\right) .
\end{aligned}
$$

To estimate the three sums above, we use an argument somewhat similar to the one used for estimating the right hand side of (18) (see estimates (20) and (21)).

For the first sum in (26) we have, by Mertens's estimate,

$$
\sum_{P\left(d_{1}\right) \leq g(t)} \frac{1}{d_{1}} \leq \prod_{q \leq g(t)}\left(1-\frac{1}{q}\right)^{-1} \ll \log (g(t)) \ll \log \log t .
$$

For the second sum in (26) we have, by the multinomial formula, Mertens's estimate (8), and Stirling's formula,

$$
\begin{aligned}
\sum_{\substack{P(d) \leq f\left(t /(\log t)^{2}\right) \\
\omega(d)<S(t)}} \frac{1}{d} & \leq \sum_{k<S(t)} \frac{1}{k !}\left(\sum_{p \leq f\left(t /(\log t)^{2}\right)} \sum_{\alpha \geq 1} \frac{1}{p^{\alpha}}\right)^{k} \\
& \leq \sum_{k<S(t)}\left(\frac{e \log \log \left(f\left(t /(\log t)^{2}\right)\right)+c_{5}}{k}\right)^{k} \\
& \leq \sum_{k<S(t)}\left(\frac{0.25 e \log \log t+c_{5}}{k}\right)^{k}
\end{aligned}
$$

where $c_{5}>0$ is some constant. Again, from the fact that for a fixed positive value of $t$ the function $s \mapsto(e t / s)^{s}$ is increasing for all positive $s<t$, and since $S(t) \leq(12)^{-2} \log \log t<0.25 \log \log t$, it follows easily that in the last sum in (28) the last term dominates, therefore

$$
\sum_{\substack{P(d) \leq f\left(t /(\log t)^{2}\right) \\ \omega(d)<S(t)}} \frac{1}{d} \ll S(t)\left(\frac{0.25 e \log \log t+c_{5}}{S(t)}\right)^{S(t)} \ll(\log t)^{c_{6}} \log \log t,
$$

where $c_{6}:=(12)^{-2} \log (36 e)$.

As for the third sum in (26), we have, again by Mertens's estimate,

$$
\begin{aligned}
\sum_{\substack{l \leq t \\
p(l) \geq f\left(t /(\log t)^{2}\right)}} \frac{1}{l} & \leq \prod_{f\left(t /(\log t)^{2}\right) \leq q \leq t}\left(1-\frac{1}{q}\right)^{-1} \ll \frac{\log t}{\log f\left(t /(\log t)^{2}\right)} \\
& \ll(\log t)^{3 / 4} .
\end{aligned}
$$


Using estimates (27), (29) and (30) in (26), we get

$$
\# \mathcal{P}_{2}(t) \ll \frac{t(\log \log t)^{5}}{(\log t)^{5 / 4-c_{6}}} \ll \frac{t}{(\log t)^{6 / 5}},
$$

because $c_{6}<1 / 20$. Thus, by (23) and (26), we get

$$
\# \mathcal{P}(t) \leq \# \mathcal{P}_{1}(t)+\# \mathcal{P}_{2}(t) \ll \frac{t}{(\log t)^{6 / 5}} .
$$

Hence, the sum of the reciprocals of the members of $\mathcal{P}$ is convergent, and in fact, by partial summation, from estimate (31) one gets immediately

$$
\sum_{\substack{p>t \\ p \in \mathcal{P}}} \frac{1}{p} \ll \frac{1}{(\log t)^{1 / 5}} .
$$

We now let

$$
\mathcal{C}_{2}:=\{n \leq x: p \mid n \text { for some prime } p>z \text { in } \mathcal{P}\} .
$$

To bound $\# \mathcal{C}_{2}$, let $p>z$ be a fixed prime in $\mathcal{P}$. The number of $n \leq x$ which are multiples of $p$ is at most $x / p$. Hence,

$$
\# \mathcal{C}_{2} \leq \sum_{\substack{p>z \\ p \in \mathcal{P}}} \frac{x}{p} \leq x \sum_{\substack{p>z \\ p \in \mathcal{P}}} \frac{1}{p} \ll \frac{x}{(\log z)^{1 / 5}}=\frac{x}{(\log x)^{1 / 10}},
$$

where we have used the bound (33) with $t=z$.

We now show that

$$
\mathcal{C} \subset \mathcal{C}_{1} \cup \mathcal{C}_{2}
$$

for large values of $x$. We recall that $\mathcal{I}=\left[v^{2}, w\right)$. Indeed, assume that $n \leq x$ is neither in $\bigcup_{i=1}^{4} \mathcal{S}_{i}$ nor in $\mathcal{C}_{1} \cup \mathcal{C}_{2}$. Then, since $n \notin \mathcal{C}_{1}$, there exist at least $R$ prime factors of $n$ which are $>z$. Let $z<p_{1}<\cdots<p_{R}$ be $R$ such primes. Since $z>v$ and $n \notin \mathcal{S}_{3}$, it follows that $p_{i} \| n$ for $i=1, \ldots, R$, therefore $\sigma(n)$ is a multiple of $\prod_{i=1}^{R}\left(p_{i}+1\right)$. Since $p_{i}>z$, it follows that $f\left(p_{i}\right) \leq f(x)=w$ and $g\left(p_{i}\right)>g(z)=v^{2}=(\log x)^{4}$. Hence, $\mathcal{I}\left(p_{i}\right) \subset \mathcal{I}$. Put

$$
T=\left\lfloor(24)^{-2} \log \log x\right\rfloor<S(z) .
$$

Since $n \notin \mathcal{C}_{2}$, it follows that

$$
\omega_{\mathcal{I}}\left(p_{i}+1\right) \geq \omega_{\mathcal{I}\left(p_{i}\right)}\left(p_{i}+1\right) \geq S\left(p_{i}\right)>S(z)>T
$$

for all $i=1, \ldots, R$. Since $n \notin \mathcal{S}_{4}$ (more precisely, since $n \notin \mathcal{B}_{3}$ ), we know that there exists no prime $q>v^{2}$ such that $q^{2} \mid \sigma(n)$. Thus,

$$
\omega_{\mathcal{I}}(\sigma(n)) \geq \sum_{i=1}^{R}\left(\omega_{\mathcal{I}}\left(p_{i}+1\right)\right)>T R>N,
$$


which completes the proof of the inclusion (35). It is now clear that (35) together with the bounds (22) and (34) shows that if we put

(36) $\mathcal{S}_{5}:=\left\{n \leq x: n \notin \bigcup_{i=1}^{4} \mathcal{S}_{i}\right.$ and either $n$ or $n+1$ belongs to $\left.\mathcal{C} \cup 2 \mathcal{C}\right\}$, then

$$
\# \mathcal{S}_{5} \leq 2 \# \mathcal{C} \leq 2 \# \mathcal{S}_{1}+2 \# \mathcal{S}_{2} \ll x /(\log x)^{1 / 10} .
$$

From now on, we assume that $n \notin \bigcup_{i=1}^{5} \mathcal{S}_{i}$.

2.6. Eliminating integers $n$ such that $\sigma(n)$ and $\sigma(n+1)$ have a large common prime factor. We put

$$
\begin{aligned}
\mathcal{S}_{6}:=\left\{n \leq x: n \notin \bigcup_{i=1}^{5} \mathcal{S}_{i} \text { and } q \mid \operatorname{gcd}(\sigma(n), \sigma(n+1))\right. & \\
& \text { for some prime } q \in \mathcal{I}\} .
\end{aligned}
$$

Let $n \in \mathcal{S}_{6}$ and suppose that $q \in \mathcal{I}$ is a common factor of both $\sigma(n)$ and $\sigma(n+1)$. Since $q \geq v^{2}$ and $n$ is neither in $\mathcal{S}_{4}$ (more precisely, $n \notin \mathcal{B}_{3}$ ), nor in $\mathcal{S}_{3}$, there exist prime factors $p_{0}$ and $p_{1}$ of $n$ and $n+1$ respectively (necessarily distinct) such that $p_{i} \| n+i$ and $q \mid p_{i}+1$ for $i=0,1$. Let $\mathcal{S}_{6,1}$ be the set of $n \in \mathcal{S}_{6}$ such that $p_{0} p_{1} \leq x$. Then, given $p_{0}$ and $p_{1}$, we have $n \equiv-i\left(\bmod p_{i}\right)$ for $i=0,1$, which puts $n$ into a fixed arithmetic progression modulo $p_{0} p_{1}$. The number of such $n \leq x$ is at most $x /\left(p_{0} p_{1}\right)+1 \leq 2 x /\left(p_{0} p_{1}\right)$ (because $\left.p_{0} p_{1} \leq x\right)$. Thus,

$$
\begin{aligned}
\# \mathcal{S}_{6,1} & \leq \sum_{q \in \mathcal{I}} \sum_{\substack{p_{i} \leq x \\
p_{i} \equiv-1(\bmod q), i=0,1}} \frac{x}{p_{0} p_{1}} \leq x \sum_{q \in \mathcal{I}} \frac{1}{2}\left(\sum_{\substack{p \leq x \\
p \equiv-1(\bmod q)}} \frac{1}{p}\right)^{2} \\
& \ll x(\log \log x)^{2} \sum_{q \geq v^{2}} \frac{1}{q^{2}} \ll \frac{x(\log \log x)^{2}}{v^{2}}=\frac{x(\log \log x)^{2}}{(\log x)^{8}} .
\end{aligned}
$$

In the above estimates, we used the bound (9) together with the fact that $p_{q-1, q} \geq 2 q-1$.

Now let

$$
\mathcal{D}:=\left\{n \leq x: n \notin \bigcup_{i=1}^{5} \mathcal{S}_{i} \text { and } n=p m \text { for some } m \leq w \text { and some prime } p\right\} .
$$

Since $n \geq x /(\log x)^{2}$ (because $\left.n \notin \mathcal{S}_{1}\right)$, we get

$$
p=\frac{n}{m} \geq \frac{x}{w(\log x)^{2}}>x^{1 / 2}
$$


for large $x$. Fix $m$. Then the number of possibilities for $p \leq x / m$ does not exceed

$$
\pi\left(\frac{x}{m}\right) \ll \frac{x}{m \log (x / m)} \ll \frac{x}{m \log x},
$$

where we have used the fact that $x / m>p>x^{1 / 2}$. Thus, summing up over all the admissible values of $m$, we get

$$
\# \mathcal{D} \leq \sum_{m \leq w} \frac{x}{m \log x}=\frac{x}{\log x} \sum_{m \leq w} \frac{1}{m} \ll \frac{x \log w}{\log x}=\frac{x}{(\log x)^{3 / 4}}
$$

Hence, if we put

$$
\mathcal{S}_{6,2}:=\mathcal{S}_{6} \cap\{n \leq x: \text { either } n \text { or } n+1 \text { belongs to } \mathcal{D}\},
$$

then

$$
\# \mathcal{S}_{6,2} \leq 2 \# \mathcal{D} \ll x /(\log x)^{3 / 4}
$$

Now let

$$
\mathcal{E}:=\left\{n \leq x: n \notin \bigcup_{i=1}^{5} \mathcal{S}_{i} \text { and } q \mid \operatorname{gcd}(n, \sigma(n)) \text { for some prime } q \geq v^{2}\right\} .
$$

We now estimate the cardinality of $\mathcal{E}$. Assume that $q \geq v^{2}$ is such that $q \mid \operatorname{gcd}(n, \sigma(n))$ for some $n \notin \bigcup_{i=1}^{5} \mathcal{S}_{i}$. Since $n \notin \mathcal{S}_{2} \cup \mathcal{S}_{3}$, we have $q \mid p+1$ for some prime factor $p$ of $n$. Fix $p$. Then the number of $n \leq x$ which are multiples of both $p$ and $q$ does not exceed $x /(p q)$. Thus,

$$
\begin{aligned}
\# \mathcal{E} & \leq \sum_{q \geq v^{2}} \sum_{\substack{p \leq x \\
p \equiv-1(\bmod q)}} \frac{x}{p q} \leq x \sum_{q \geq v^{2}} \frac{1}{q} \sum_{\substack{p \leq x \\
p \equiv-1(\bmod q)}} \frac{1}{p} \\
& \ll x \log \log x \sum_{q \geq v^{2}} \frac{1}{q^{2}} \ll \frac{x \log \log x}{(\log x)^{8}}
\end{aligned}
$$

where in the above estimates we have used again the bound (9). Thus, if we write

$$
\mathcal{S}_{6,3}:=\mathcal{S}_{6} \cap\{n \leq x \text { : either } n \text { or } n+1 \text { belongs to } \mathcal{E}\},
$$

then we get

$$
\# \mathcal{S}_{6,3} \leq 2 \# \mathcal{E} \ll \frac{x(\log \log x)}{(\log x)^{8}} .
$$

For the remaining numbers $n$ in $\mathcal{S}_{6} \backslash \bigcup_{i=1}^{3} \mathcal{S}_{6, i}$, we write $n=p_{0} a$ and $n+1=p_{1} b$. Note that both $a$ and $b$ exceed $q$ because $n \notin \mathcal{D}$. We now let $\mathcal{S}_{6,4}$ be the subset of $\mathcal{S}_{6} \backslash \bigcup_{i=1}^{3} \mathcal{S}_{6, i}$ such that $a b \leq x / q$. Fix the prime $q$. Since $p_{1} b-p_{0} a=1$, and $p_{0}$ and $p_{1}$ are both congruent to -1 modulo $q$, we get $b-a \equiv-1(\bmod q)$. Fix two coprime positive integers $a$ and $b$ 
with $a b \leq x / q$ such that $b-a \equiv-1(\bmod q)$. It suffices to find an upper bound for the number of choices of $p_{0}$, since once $p_{0}$ is known, then $n$ is also known. Since $b \mid p_{0} a+1$, it follows that $p_{0}$ is in a certain arithmetic progression modulo $b$. Further, $p_{0} \equiv-1(\bmod q)$. Note that $q$ does not divide $b$, otherwise $q \mid \operatorname{gcd}(n+1, \sigma(n+1))$, which is impossible since $n+1 \notin \mathcal{E}$. Thus, we can apply the Chinese remainder theorem and conclude that $p_{0}$ is in a certain arithmetic progression modulo $b q$. Since $p_{0}=n / a \leq x / a$, the number of such $p_{0}$ is (even forgetting the condition that $p_{0}$ is prime) at most $x /(a b q)+1 \leq 2 x /(a b q)$ (here, we use the fact that $a b q \leq x)$. Summing up over all such $a, b$ and $q$, we get

$$
\begin{aligned}
\# \mathcal{S}_{6,4} & \ll \sum_{q \in \mathcal{I}} \sum_{a \leq x / q} \sum_{\substack{q<b \leq x /(a q) \\
b \equiv a-1(\bmod q)}} \frac{x}{a b q} \\
& \leq x \sum_{q \in \mathcal{I}} \sum_{a<x / q} \frac{1}{a q} \sum_{\substack{q<b \leq x /(a q) \\
b \equiv a-1(\bmod q)}} \frac{1}{b} \leq x \sum_{q \in \mathcal{I}} \sum_{a \leq x / q} \frac{\log x}{a q^{2}} \\
& \leq x(\log x)\left(\sum_{a \leq x} \frac{1}{a}\right)\left(\sum_{q \geq v^{2}} \frac{1}{q^{2}}\right) \ll \frac{x(\log x)^{2}}{v^{2}}=\frac{x}{(\log x)^{6}} .
\end{aligned}
$$

Finally, let $\mathcal{S}_{6,5}:=\mathcal{S}_{6} \backslash \bigcup_{i=1}^{4} \mathcal{S}_{6, i}$. With the previous notations, we have $p_{0} p_{1}>x$, therefore $\max \left\{p_{0}, p_{1}\right\}>x^{1 / 2}$. Assume that $p_{0}>p_{1}$, since the other case is entirely similar. Thus, $p_{0}=P(n)$. Since $a b \geq x / q$, we get $p_{0} p_{1}=$ $n(n+1) /(a b) \leq x(x+1) /(a b) \leq 2 x q$. Fix the number $p_{1}$. Then $p_{0}>x^{1 / 2}$ is a prime number $\leq 2 x q / p_{1}$ which is in a fixed arithmetic progression modulo $q$ (namely, is congruent to $p_{1}$ modulo $q$ ). The number of such numbers is, by the Brun-Titchmarsh theorem,

$$
\ll \frac{x q}{p_{1} \phi(q) \log \left(x / p_{1}\right)} \ll \frac{x}{p_{1} \log x^{1 / 3}} \ll \frac{x}{\log x},
$$

where we have used the fact that $x / p_{1} \geq p_{0} /(2 q)>x^{1 / 2} /(2 w)>x^{1 / 3}$ for large values of $x$. Thus, summing up over all the possible values of $q$ and $p_{1}$, we get

$$
\begin{aligned}
\# \mathcal{S}_{6,5} & \leq \frac{x}{\log x} \sum_{q \leq w} \sum_{\substack{p_{1} \leq x \\
p_{1} \equiv-1(\bmod q)}} \frac{1}{p_{1}} \\
& =\frac{x}{\log x} \sum_{p_{1} \leq x} \frac{\omega\left(p_{1}+1\right)}{p_{1}} \leq \frac{x K}{\log x} \sum_{p_{1} \leq x} \frac{1}{p_{1}} \ll \frac{x(\log \log x)^{2}}{\log x},
\end{aligned}
$$

where we changed the order of summation and used Mertens's estimate (8) as well as the fact that $p_{1}$ is a prime factor of $n+1 \notin \mathcal{B}_{2} \subset \mathcal{S}_{4}$. 
Since the sets $\mathcal{S}_{6, i}$ for $i=1, \ldots, 5$ cover $\mathcal{S}_{6}$, we deduce from estimates (39), (40), (42), (43) and (44) that

$$
\# \mathcal{S}_{6} \leq \sum_{i=1}^{5} \# \mathcal{S}_{6, i} \ll x /(\log x)^{3 / 4} .
$$

From now on, we assume that $n \leq x$ is not in $\bigcup_{i=1}^{6} \mathcal{S}_{i}$.

2.7. Analyzing the remaining set $\mathcal{T} \backslash \bigcup_{i=1}^{6} \mathcal{S}_{i}$. Put $\mathcal{S}_{7}:=\mathcal{S}(x) \backslash \bigcup_{i=1}^{6} \mathcal{S}_{i}$ and let $n \in \mathcal{S}_{7}$. Then we may write $n=P a, n+1=Q b$, where $P=P(n)$ and $Q=P(n+1)$. We assume that $a$ is odd since the other case can be dealt with analogously. Since $n$ is odd, we have $n \mid T_{n}$. Furthermore, since $n$ and $(n+1) / 2$ are coprime, and since $P \| n$ (because $y>v$ holds for large $x$ and $\left.n \notin \mathcal{S}_{1} \cup \mathcal{S}_{2}\right)$, we conclude that $\sigma\left(T_{n}\right)=(P+1) \sigma(a) \sigma((n+1) / 2)$.

Now let $m$ be such that $\sigma\left(T_{n}\right)=m(m+1)$. Since $\sigma(k) \ll k \log \log k$ for all positive integers $k$, we get

$$
m^{2}<\sigma\left(T_{n}\right) \ll T_{n} \log \log T_{n} \ll x^{2} \log \log x,
$$

therefore

$$
m \leq c_{7} x(\log \log x)^{1 / 2}
$$

with some positive constant $c_{7}$. We write $X:=c_{7} x(\log \log x)^{1 / 2}$. Furthermore,

$$
(m+1)^{2}>\sigma\left(T_{n}\right)>T_{n} \gg n^{2} \geq\left(\frac{x}{(\log x)^{2}}\right)^{2}
$$

(because $\left.n \notin \mathcal{S}_{1}\right)$, therefore $m \gg x /(\log x)^{2}$.

We now fix the number $a$. Let $\mathcal{M}(a)$ be the set of all acceptable values of $m$, i.e., the set of all positive integers $m$ such that $m(m+1)=\sigma\left(T_{n}\right)$ for some $n$ of the form $n=P a \leq x$ with $P=P(n)$ and not in $\bigcup_{i=1}^{6} \mathcal{S}_{i}$. Then, when $a$ is fixed, $n$ is determined by its largest prime factor $P$. Since $(P+1) \mid \sigma\left(T_{n}\right)$, we infer that $P+1$ is a divisor of $m(m+1)$ for some $m \in$ $\mathcal{M}(a)$. Thus, the number of possibilities for $P$ when $a$ and $m \in \mathcal{M}(a)$ are fixed does not exceed $\tau(m(m+1))=\tau(\sigma(n(n+1) / 2))<\exp \left(8 K^{2}\right)$, because $n \notin \mathcal{S}_{4}$.

Hence, for a fixed $a$, the number of possibilities for $n$ is

$$
\sum_{m \in \mathcal{M}(a)} \tau(m(m+1)) \leq \exp \left(8 K^{2}\right) \# \mathcal{M}(a)
$$

It remains to estimate $\# \mathcal{M}(a)$. Note that if $m \in \mathcal{M}(a)$, then $\sigma(a)$ divides $m(m+1)$. Thus, there exists a decomposition of the form $\sigma(a)=d_{1} d_{2}$, where $\operatorname{gcd}\left(d_{1}, d_{2}\right)=1$, such that $d_{1} \mid m$ and $d_{2} \mid m+1$. The number of such decompositions of $\sigma(a)$ is $\leq \tau(\sigma(a)) \leq \tau(\sigma(n))<\exp \left(4 K^{2}\right)$. Fix the pair $\left(d_{1}, d_{2}\right)$. Then $m=d_{1} \gamma$ and $m+1=d_{2} \delta$, so $d_{2} \mid d_{1} \gamma+1$, which puts $\gamma$ into a certain arithmetic progression modulo $d_{2}$. Let $r_{2}>0$ be the smallest 
member of this progression. Clearly, $r_{2} \leq d_{2}$. In fact, even the inequality $r_{2}<d_{2}$ holds if $d_{2}>1$, because the relation $r_{2}=d_{2}$ leads to $d_{2} \mid d_{1} r_{2}+1$; hence, $d_{2}=1$.

Thus $m=d_{1}\left(d_{2} \lambda+r_{2}\right)$, where $\lambda \geq 0$ is an integer. Further, $m+1=$ $d_{2}\left(d_{1} \lambda+r_{1}\right)$, where we use $r_{1}=\left(d_{1} r_{2}+1\right) / d_{2}$. The relation $\sigma\left(T_{n}\right)=m(m+1)$ now simplifies to

$$
(P+1) \sigma((n+1) / 2)=\left(d_{1} \lambda+r_{1}\right)\left(d_{2} \lambda+r_{2}\right) .
$$

It remains to count such values of $\lambda$. Note first that $d_{1} d_{2} \lambda \leq m \leq X$, therefore

$$
\lambda \leq \frac{X}{d_{1} d_{2}}=\frac{X}{\sigma(a)} \ll \frac{x(\log \log x)^{1 / 2}}{a} .
$$

Further, $d_{1} d_{2}(\lambda+1)>m \gg x /(\log x)^{2}$, therefore

$$
\begin{aligned}
\lambda+1 & \gg \frac{x}{\left(d_{1} d_{2}\right)(\log x)^{2}}>\frac{x}{\sigma(a)(\log x)^{2}} \gg \frac{x}{a} \frac{1}{(\log x)^{2}(\log \log x)} \\
& >\frac{P}{(\log x)^{3}}>y^{2 / 3}
\end{aligned}
$$

for large values of $x$. Hence, we may assume that $x$ is so large that $\lambda>\sqrt{y}$.

Let $\Lambda_{1}:=d_{1} \lambda+r_{1}$ and $\Lambda_{2}:=d_{2} \lambda+r_{2}$. Note that $\Lambda_{2} \mid m$ and $\Lambda_{1} \mid m+1$, so they are coprime. Since $n \notin \mathcal{S}_{5}$, it follows that $\sigma((n+1) / 2)$ has at least $N$ distinct prime factors in $\mathcal{I}$. Thus, at least one of $\Lambda_{1}$ or $\Lambda_{2}$ has at least $N / 2$ distinct prime factors in $\mathcal{I}$. Assume that $\omega_{\mathcal{I}}\left(\Lambda_{1}\right) \geq N / 2$. Put $U:=\lfloor N / 2\rfloor$. Let $\mathcal{L}$ be the set of all squarefree positive integers $l$ such that $p(l) \geq v^{2}$, $P(l) \leq w$ and $\omega(l)=U$. Then

$$
\begin{aligned}
l \leq w^{U} & =\exp \left(O\left((\log x)^{1 / 4}(\log \log x)^{2}\right)\right) \\
& =o(\sqrt{y})=o(\lambda) \quad \text { for all } l \in \mathcal{L}
\end{aligned}
$$

as $x \rightarrow \infty$. Further, for each $\lambda$ there exists $l \in \mathcal{L}$ such that $d_{1} \lambda+r_{1} \equiv 0$ $(\bmod l)$. Note that $l$ is coprime to $d_{1}$, since if there exists $q \mid \operatorname{gcd}\left(l, d_{1}\right)$, we then deduce that, on the one hand, $q|\sigma(a)| \sigma(n)$, and on the other hand $q\left|d_{1} \lambda+r_{1}\right|(P+1) \sigma((n+1) / 2)$, therefore either $q \mid P+1$ (which is impossible since $\left.n \notin \mathcal{B}_{3} \subset \mathcal{S}_{4}\right)$, or $q \mid \sigma((n+1) / 2)$ (which is impossible because $n \notin \mathcal{S}_{6}$ ). Thus, the congruence $d_{1} \lambda+r_{1} \equiv 0(\bmod l)$ puts $\lambda \leq X /\left(d_{1} d_{2}\right)$ in a certain residue class modulo $l$. The number of such values is at most $\leq X /\left(d_{1} d_{2} l\right)+$ $1 \leq 2 X /\left(d_{1} d_{2} l\right)$ (here, we use the observation that $X /\left(d_{1} d_{2}\right)>\sqrt{y}>l$, which follows for $x$ large from (47)). Summing up over all the possible values of $l \in \mathcal{L}$ and over all the possible factorizations $d_{1} d_{2}=\sigma(a)$, we get

$$
\# \mathcal{M}(a) \leq \exp \left(4 K^{2}\right) \frac{X}{\sigma(a)} \sum_{l \in \mathcal{L}} \frac{1}{l} \ll \exp \left(4 K^{2}\right) \frac{x(\log \log x)^{1 / 2}}{a} \sum_{l \in \mathcal{L}} \frac{1}{l} .
$$


Summing up over $a \leq x$ and using estimate (46), we get

$$
\begin{aligned}
\# \mathcal{S}_{7} & \leq \exp \left(8 K^{2}\right) \sum_{a \leq x} \# \mathcal{M}(a) \\
& \ll x \exp \left(12 K^{2}\right)(\log \log x)^{1 / 2}\left(\sum_{a \leq x} \frac{1}{a}\right)\left(\sum_{l \in \mathcal{L}} \frac{1}{l}\right) .
\end{aligned}
$$

It remains to estimate the sum of the reciprocals of the members of $\mathcal{L}$. But by the familiar procedure of using the multinomial formula, Mertens's estimate (8), and Stirling's formula,

$$
\begin{aligned}
\sum_{l \in \mathcal{L}} \frac{1}{l} & \leq \frac{1}{U !}\left(\sum_{v^{2} \leq q \leq w} \frac{1}{q}\right)^{U} \leq\left(\frac{e \log \log w+O(1)}{U}\right)^{U} \\
& \leq \exp \left(-c_{8} K^{2} \log K\right)
\end{aligned}
$$

for some positive constant $c_{8}$, where we use the fact that $U \gg K$ and $\log \log w<\log \log x \ll \sqrt{U}$. Hence, inserting (49) into (48), we get

$$
\# \mathcal{S}_{7} \ll x \frac{\exp \left(12 K^{2}\right)(\log x)(\log \log x)^{1 / 2}}{\exp \left(c_{8} K^{2} \log K\right)}<\frac{x}{(\log x)^{2}}
$$

for large values of $x$.

The conclusion of Theorem 1 follows from the fact that $\mathcal{S}_{i}$ cover $\mathcal{S}$ for $i=1, \ldots, 7$ together with the estimates (3), (5), (7), (16), (37), (45) and (50).

3. The general case. Let us first address the case when $\operatorname{deg} f=1$. Assume first that $\operatorname{deg} g>1$. In this case, since $\sigma(|f(n)|) \ll x \log \log x$ for all $n \leq x$, it follows easily that the cardinality of the set of values of

$$
\left\{\sigma(|f(n)|): n \in \mathcal{S}_{f, g}(x)\right\}
$$

is $\ll(x \log \log x)^{1 / 2}=x^{1 / 2+o(1)}$. Assume now that the value of $\sigma(|f(n)|)$ is given and let us count how many of our $n$ 's could have given rise to this value. From an immediate adaptation of the results from Section 2.4, we may assume that $\tau(\sigma(|f(n)|)) \leq \exp \left(4 K^{2}\right)$. Now if $p^{\alpha} \| f(n)$, then $\left(p^{\alpha}-1\right) /(p-1)$ is a divisor of $\sigma(|f(n)|)$, and this can be chosen in at most $\exp \left(4 K^{2}\right)$ ways. Given $\left(p^{\alpha}-1\right) /(p-1)$, since $\alpha \ll \log x$, it follows that the number $p^{\alpha}$ is determined in at most $\log x$ ways. Thus, there are at most $(\log x) \exp \left(4 K^{2}\right) \leq$ $\exp \left(5 K^{2}\right)$ ways of choosing the prime power $p^{\alpha}$ once $\sigma(|f(n)|)$ is known. Since at most $K$ values for $p^{\alpha}$ determine $n$, the number of possibilities for $n$ once $\sigma(|f(n)|)$ is known is at most $\exp \left(5 K^{3}\right)=x^{o(1)}$. So, the result of Theorem 1 is valid in this case as well.

Assume now that $\operatorname{deg} f=\operatorname{deg} g=1$. In this last case, we may assume that $g(X):=a X+b$ for some integers $a>0$ and $b$. Let $c:=\operatorname{gcd}(a, b)$, and write $a=a_{1} c$ and $b=b_{1} c$. Thus, $g(x)=c\left(a_{1} X+b_{1}\right)$, where $a_{1}$ and $b_{1}$ are 
coprime. If $a_{1}=1$, then both $\mathcal{F}_{f, g}(x)$ and $\mathcal{S}_{f, g}(x)$ contain almost all positive integers up to $x$, so they are of cardinalities $x+o(x)$ as $x \rightarrow \infty$. Finally, if $a_{1}>1$ and $n \in \mathcal{F}_{f, g}$, then $f(n)$ must have a bounded number of prime factors $p \equiv 1\left(\bmod a_{1}\right)$ in its factorization, and sieve methods lead to the conclusion that

$$
\# \mathcal{F}_{f, g}(x) \ll \frac{x}{(\log x)^{1 / \phi\left(a_{1}\right)+o(1)}} \quad \text { as } x \rightarrow \infty .
$$

It is not hard to show that the above upper bound is also a lower bound on the cardinality $\# \mathcal{F}_{f, g}(x)$, so Theorem 1 does not apply say if $\phi\left(a_{1}\right)>10$. A similar argument applies to numbers $n \in \mathcal{S}_{f, g}$ which, in particular, must have only a bounded number of prime factors $p \equiv-1\left(\bmod a_{1}\right)$ which appear with exponent 1 in their prime factorization.

We mention that several particular instances of this problem for the case when $\operatorname{deg} f=1$ were investigated previously, e.g. in [1] where the $\operatorname{set} \mathcal{F}_{f, g}(x)$ was analyzed for $f(X)=X$ and $g(X)=X^{2}$.

We now return to the case when $\operatorname{deg} f>1$. We only deal with $\mathcal{S}_{f, g}(x)$ for the general case. One starts by writing

$$
f(X):=\prod_{i=1}^{d} L_{i}(X),
$$

where $L_{i}(X):=a_{i} X+b_{i}$ with $a_{i}>0$, and $-b_{i} / a_{i} \neq-b_{j} / a_{j}$ for $i \neq j$. By putting $n$ into a fixed progression modulo the least common denominator of the $a_{i}$ 's and $b_{i}$ 's, if needed, one may assume that $a_{i}$ and $b_{i}$ are integers. Now one proceeds in the same way as in the proof for the particular case by discarding integers $n \leq x$ which are small, or for which $L_{i}(n)$ is smooth for some $i=1, \ldots, d$, or for which $L_{i}(n)$ has a large powerful divisor, or for which $\sigma\left(L_{i}(n)\right)$ has too many divisors, or too few prime factors in $\mathcal{I}$ for some $i=1, \ldots, d$, or for which $\sigma\left(L_{i}(n)\right)$ and $\sigma\left(L_{j}(n)\right)$ have a common prime factor in $\mathcal{I}$ for some $i \neq j$. For the remaining numbers $n \leq x$, one fixes $a=L_{1}(n) / P\left(L_{1}(n)\right)$ and notices that if $m$ is fixed, then $P\left(L_{1}(n)\right)+1$ is fixed in $\tau(g(m))=\exp \left(O\left(K^{2}\right)\right)$ ways. It remains to count the number of possibilities for $m$. Clearly, $m \leq X$, where $X$ was defined in Section 2.7. Now one distinguishes three cases:

CASE 1: $\operatorname{deg} g=d$ and $g(X)$ has no double root. In this case, if further $g(X):=\prod_{i=1}^{d} M_{i}(X)$ for some linear forms $M_{i}(X)$ for $i=1, \ldots, d$, then one can proceed as in the particular case when $g(X)=X(X+1)$. But the condition that $g(X) \in \mathbb{Q}(X)$ factors in linear forms is not needed. Indeed, as in the particular case when $f(X)=X(X+1) / 2$, let us also fix a divisor $l \in \mathcal{L}$ of $\sigma\left(L_{2}(n)\right)$. As before, $\sigma(a)$ and $l$ are coprime and $\sigma(a) l<X$. Now the congruence $g(m) \equiv 0(\bmod \sigma(a) l)$ puts $m \leq X$ into $O\left(d^{\omega(\sigma(a) l)}\right)=$ $\exp \left(O\left(K^{2}\right)\right)$ residue classes modulo $\sigma(a) l$ (because $g$ has only simple roots). 
For each of these progressions, the number of acceptable values for $m$ is $\leq X /(\sigma(a) l)+1 \leq 2 X /(\sigma(a) l)$. Summing up over all the acceptable values for $a$ and $l$ we deduce, as in Section 2.7, that the number of acceptable values for $m$ is $x \exp \left(-c_{8} K^{2} \log K\right)$, which leads to the desired conclusion.

CASE 2: $\operatorname{deg} g=d$ but $g(X)$ has a double root. In this case, we further shrink the set of allowable $n \leq x$ by also removing those positive integers $n$ such that $L_{i}(n) \in \mathcal{S}_{8}$, where

$$
\mathcal{S}_{8}:=\left\{n \leq x: q^{2} \mid \sigma(n) \text { for some } q>(\log x)^{1 / 4}\right\} .
$$

Suppose first that $n \leq x$ has a powerful divisor $>(\log x)^{1 / 5}$. Then, as in Section 2.3, the number of such $n$ is

$$
\leq x \sum_{\substack{a>(\log x)^{1 / 5} \\ a \text { powerful }}} \frac{1}{a} \ll \frac{x}{(\log x)^{1 / 10}} .
$$

Assume now the largest powerful divisor of $n$ is $\leq(\log x)^{1 / 5}$. Write $n=n_{0} n_{1}$, where $n_{0}$ and $n_{1}$ are coprime, $n_{0}$ is powerful, and $n_{1}$ is squarefree. Then

$$
\sigma(n)=\sigma\left(n_{0}\right) \sigma\left(n_{1}\right),
$$

and $\sigma\left(n_{0}\right) \ll n_{0} \log \log n_{0} \ll(\log x)^{1 / 5} \log \log \log x=o\left((\log x)^{1 / 4}\right)$. Thus, if such $n$ is in $\mathcal{S}_{8}$ and $x$ is large, it follows that there exists a prime $q>(\log x)^{1 / 4}$ such that $q^{2} \mid \sigma\left(n_{1}\right)$. As in Section 2.4, the number of such $n$ is

$$
\leq x \sum_{q>(\log x)^{1 / 4}} \sum_{\substack{p \leq x \\ p \equiv-1\left(\bmod q^{2}\right)}} \frac{1}{p}+\sum_{q>(\log x)^{1 / 4}} \sum_{\substack{p_{1}<p_{2} \leq x \\ p_{i} \equiv-1(\bmod q)}} \frac{1}{p_{1} p_{2}},
$$

which, by arguments similar to the ones used in Section 2.4, is

$$
O\left(\frac{x(\log \log x)^{2}}{(\log x)^{1 / 4}}\right)=o\left(\frac{x}{(\log x)^{1 / 10}}\right) .
$$

Thus, $\# \mathcal{S}_{8}=o\left(x /(\log x)^{1 / 10}\right)$.

Hence, from now on we assume that $L_{i}(n) \notin \mathcal{S}_{8}$ for $i=1, \ldots, d$. Let $g_{1}(X)$ be some irreducible factor of $g(X)$ of positive degree such that $g_{1}(X)^{2} \mid g(X)$. Then $g_{1}(m)^{2} \mid \sigma(f(n))=\prod_{i=1}^{d} \sigma\left(L_{i}(n)\right)$, therefore $g_{1}(m)$ has the property that $P\left(g_{1}(m)\right)<(\log x)^{1 / 4}$. Further, since $n \notin \mathcal{S}_{3} \cup \mathcal{S}_{4}$, it follows easily that the exponent with which a prime $q$ can appear in $g_{1}(m)$ is $O\left((\log \log x)^{2}\right)$. Hence, the number of values for $g_{1}(m)$ is

$$
\leq\left(c_{9}(\log \log x)^{2}\right)^{\pi\left((\log x)^{1 / 4}\right)}=\exp \left(o(\log x)^{1 / 4}\right)=x^{o(1)} .
$$

Since for every fixed value of $g_{1}(m)$, the number $m$ can take at most $\operatorname{deg} g_{1} \leq$ $d / 2$ values, we see that the number of acceptable values for $m$ is $x^{o(1)}$, which is better (smaller) than in Case 1. 
CASE 3: $\operatorname{deg} g=d_{1}>d$. Here, the situation is even simpler. Indeed, the previous arguments imply easily that if $n \notin \mathcal{S}_{3} \cup \mathcal{S}_{4}$, then

$$
\Omega(n) \ll K^{3} \text {. }
$$

Thus, in this case $\Omega(\sigma(f(n)))=O\left(K^{3}\right)$, while $\tau(\sigma(f(n)))=\exp \left(O\left(K^{2}\right)\right)$. Since the number $m$ has $g(m) \ll x^{d}$, we get $m \ll x^{d / d_{1}}$. Hence, $\sigma(f(n))$ has at most $O\left(x^{d / d_{1}}\right)$ distinct values. For each of these values, the number of possibilities for $f(n)$ is

$$
\leq \tau(\sigma(f(n)))^{\Omega(\sigma(f(n)))}=\exp \left(O\left(K^{5}\right)\right)=x^{o(1)},
$$

which together with the fact that $n$ is determined in at most $d$ ways by $f(n)$ leads to the conclusion that the number of such values of $n$ does not exceed $x^{d / d_{1}+o(1)}$.

Minor changes (like changing $p+1$ to $p-1$ in the definition of $\mathcal{B}_{2}$ ) apply for the case of $\mathcal{F}_{f, g}(x)$. We give no further details.

REMARK. As pointed out by the referee, the proof used here has the consequence that for the polynomials corresponding to the title, $f(X)=g(X)=$ $X(X+1) / 2$, the sets $\mathcal{F}_{f, g}(x)$ and $\mathcal{S}_{f, g}(x)$ have cardinalities $O\left(x /(\log x)^{3 / 4}\right)$. Presumably, the proof can be adapted to show that the exponent $3 / 4$ can be improved to $1-\varepsilon$ for any fixed $\varepsilon>0$.

\section{References}

[1] W. D. Banks, J. B. Friedlander, C. Pomerance and I. E. Shparlinski, Multiplicative structure of values of the Euler function, in: High Primes and Misdemeanours: Lectures in Honour of the 60th Birthday of Hugh Cowie Williams, Fields Inst. Commun. 41, Amer. Math. Soc., Providence, RI, 2004, 29-47.

[2] R. R. Hall and G. Tenenbaum, Divisors, Cambridge Univ. Press, 1988.

[3] A. Ivić, The Riemann Zeta-Function. Theory and Applications, Dover, Mineola, New York, 2003.

[4] F. Luca and C. Pomerance, Irreducible radical extensions and Euler-function chains, in: Combinatorial Number Theory, Proc. of the "Integers" Conference in Honor of R. Graham's 70th Birthday, B. Landman et al. (eds.), de Gruyter, 2007, 351-362.

[5] G. Tenenbaum, Introduction to Analytic and Probabilistic Number Theory, Cambridge Univ. Press, 1995.

University of the Virgin Islands

St. Thomas, VI 00802, U.S.A.

E-mail: diannuc@uvi.edu
Instituto de Matemáticas Universidad Nacional Autonoma de México C.P. 58089, Morelia, Michoacán, México E-mail: fluca@matmor.unam.mx 